\title{
Termination rates and histological reclassification of active surveillance patients with low- and early intermediate-risk prostate cancer: results of the PREFERE trial
}

\author{
Peter Albers ${ }^{1}$ (D) $\cdot$ Thomas Wiegel $^{2} \cdot$ Heinz Schmidberger $^{3} \cdot$ Roswitha Bussar-Maatz $^{4} \cdot$ Martin Härter $^{5}$. \\ Glen Kristiansen ${ }^{6} \cdot$ Peter Martus $^{7} \cdot$ Christoph Meisner $^{7} \cdot$ Stefan Wellek $^{8} \cdot$ Klaus Grozinger $^{9} \cdot$ Peter Renner $^{10}$. \\ Martin Burmester ${ }^{11} \cdot$ Fried Schneider ${ }^{12} \cdot$ Michael Stöckle $^{13}$
}

Received: 25 November 2019 / Accepted: 2 March 2020 / Published online: 18 March 2020

(c) The Author(s) 2020

\begin{abstract}
Purpose Active surveillance (AS) strategies for patients with low- and early intermediate-risk prostate cancer are still not consistently defined. Within a controlled randomized trial, active surveillance was compared to other treatment options for patients with prostate cancer. Aim of this analysis was to report on termination rates of patients treated with AS including different grade groups.

Methods A randomized trial comparing radical prostatectomy, active surveillance, external beam radiotherapy and brachytherapy was performed from 2013 to 2016 and included 345 patients with low- and early intermediate-risk prostate cancer (ISUP grade groups 1 and 2). The trial was prematurely stopped due to slow accrual. A total of 130 patients were treated with active surveillance. Among them, 42 patients were diagnosed with intermediate-risk PCA. Reference pathology and AS quality control were performed throughout.

Results After a median follow-up time of 18.8 months, 73 out of the 130 patients (56\%) terminated active surveillance. Of these, $56(77 \%)$ patients were histologically reclassified at the time of rebiopsy, including $35 \%$ and $60 \%$ of the grade group 1 and 2 patients, respectively. No patients who underwent radical prostatectomy at the time of reclassification had radical prostatectomy specimens $\geq$ grade group 3 .

Conclusion In this prospectively analyzed subcohort of patients with AS and conventional staging within a randomized trial, the 2-year histological reclassification rates were higher than those previously reported. Active surveillance may not be based on conventional staging alone, and patients with grade group 2 cancers may be recommended for active surveillance in carefully controlled trials only.
\end{abstract}

Keywords Active surveillance $\cdot$ Prostate cancer $\cdot$ Clinical trial $\cdot$ Reclassification

\section{Introduction}

Prostate cancer $(\mathrm{PCa})$ is the most frequent cancer in men [1]. Beginning at age 40 , the likelihood of harboring prostate cancer is approximately equal to age as a percentage [2]. Approximately, half of all patients are found to have lowrisk PCa with a maximum Gleason sum (GS) score of six [ISUP grade group (GG) 1]. Based on reference pathology, in a large series of patients with radical prostatectomy and

Peter Albers

peter.albers@med.uni-duesseldorf.de

Extended author information available on the last page of the article
GG1 cancers, the rate of lymph node metastasis was zero, and the mortality rate after 20 years was $0.2 \%$ [3, 4]. Metastasis is strongly related to the Gleason pattern and expression of metastasis-related genes, but clonal evolution from Gleason pattern 3 to Gleason pattern 4 is rare [5]. Bioptic undergrading is presumably common, implying that cancers harboring a Gleason pattern 4 component at the time of the initial biopsy can be missed by systematic biopsies. Assuming more representative sampling, patients with exclusively GG1 cancers may not need immediate treatment, and active surveillance strategies may be justified. In the pivotal randomized ProtecT trial, patients with clinically localized prostate cancers were randomized between radical prostatectomy (RP), radiotherapy (RT), and active monitoring. 
Based on PSA monitoring, the 10-year cancer-specific survival rate was not different [6]. In all patients managed with active monitoring $(n=545)$, eight patients died, and in the subgroup of patients with GG1 cancers (77\%), only three deaths were reported after 10 years. However, these data are premature for survival analyses, as demonstrated by the SPCG-4 trial [7], which compared RP with watchful waiting and found follow-up data far beyond 20 years to still be highly relevant. Until now, the detailed inclusion criteria and monitoring schedules for active surveillance remain undefined, predominantly because of a lack of prospective randomized data. Furthermore, it remains unclear whether patients with GG2 cancers may also be eligible for active surveillance. In prospectively controlled trials, patients with GG2 prostate cancers were not necessarily more likely to experience reclassification than those with GG1 cancers. In a competing analysis, the rate of progression in patients with GG2 prostate cancers was higher than that of patients with GG1 cancer, but the cancer-specific survival rates were not significantly different $[8,9]$.

Based on the available data at that time, a randomized trial (PREFERE) was initiated in Germany in 2013 to prove the noninferiority of RP to RT, low-dose radiation brachytherapy (BT) and AS in patients with low- (GG1) and early intermediate-risk (GG2) prostate cancer [10]. Cancerspecific survival was defined as the primary endpoint. To facilitate patient recruitment, a preference design was used and allowed refusal of up to two trial options, as long as randomization between the remaining treatment options was accepted. Because of poor accrual, the trial was prematurely terminated in December 2016. Herein, we analyze the data of the patients randomized for and/or who chose AS in the course of this trial with a focus on the termination of AS and the histological reclassification rates with special attention to ISUP grade groups.

\section{Patients and methods}

Patients were randomized among four possible options: RP, RT, BT, or AS. Of these four options, patients could exclude up to two choices. The final treatment was based on the randomization between the remaining treatment options. The study started patient accrual in April 2013. The design of the trial and the inclusion criteria have been described elsewhere [10]. Due to slow accrual and after sample size recalculations, the inclusion criteria were changed in 2015 to include patients with GG2 cancers (see Table 1). In addition, the use of imaging techniques (MRI, C-Trus/Anna) was allowed according to published evidence, and the inclusion of all GG1 tumors, regardless of tumor extent, was allowed [11]. To further enhance accrual, patients who had obstructive

Table 1 Inclusion and exclusion criteria of the PREFERE Trial since 2015

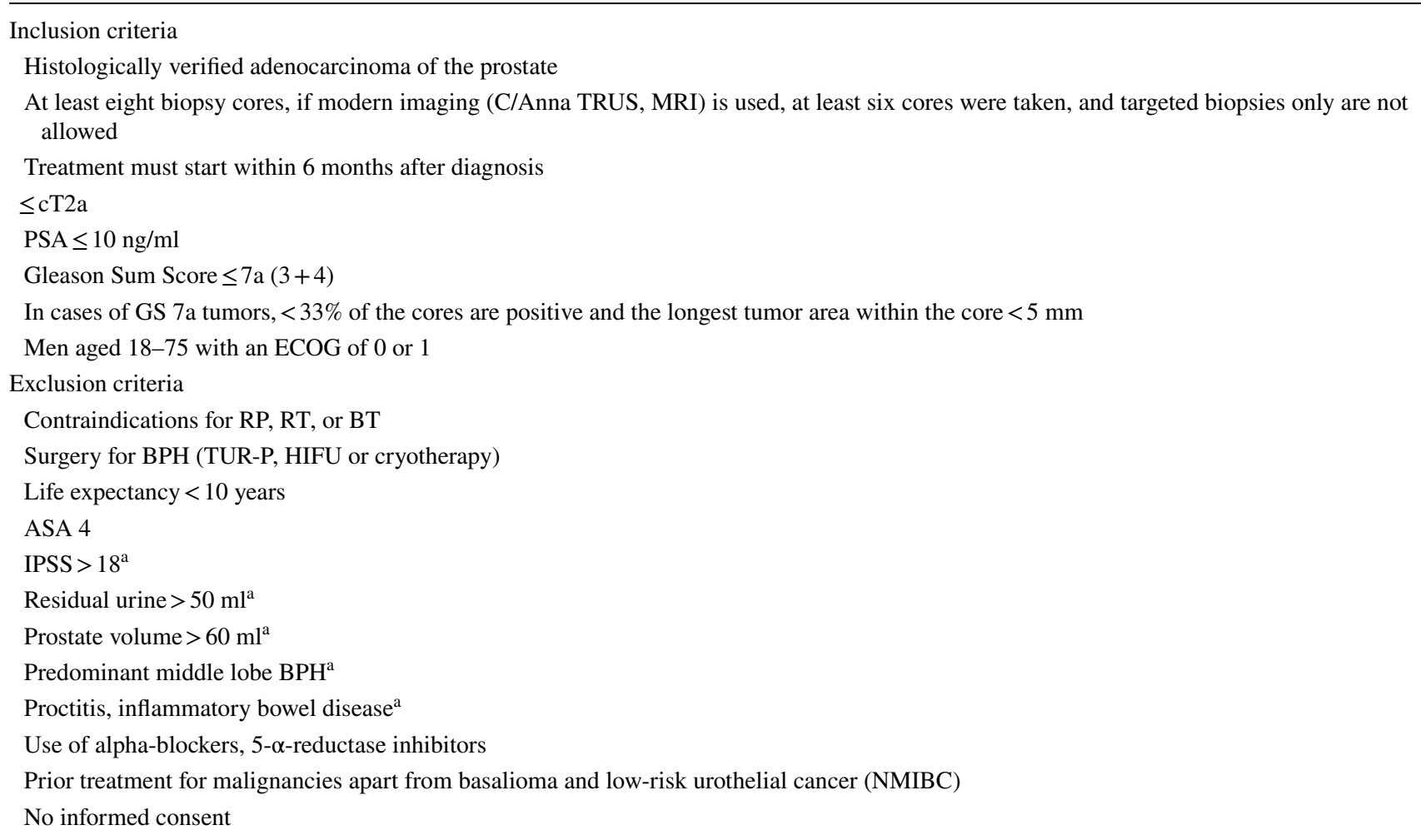

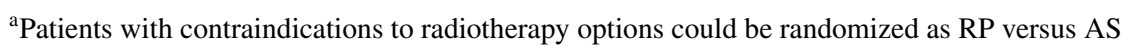


micturition disorders could be included. In December 2016, after 44 months of enrollment, the protocol was stopped for slow accrual.

The AS protocol recommended an early rebiopsy after 6 months for patients with GG2 tumors; thereafter, biopsies were recommended at 12 and 24 months. GG1 tumors had to be rebiopsied after 12 and 24 months. Thereafter, both groups had to undergo 3-years of rebiopsies until reaching 80 years of age. Clinical follow-up, including PSA values and digital rectal examination, was recommended every 3 months for the first 2 years and every 6 months thereafter.

AS was terminated if the following event occurred: (1) the patient did not wish to continue; (2) histological reclassification was observed at rebiopsy (from GG 1 to GG 2 or higher or from GG 2 to GG 3 or higher); (3) the tumor volume of GG2 tumors exceeded $\geq 33 \%$ of the biopsy cores; or (4) histological reclassification to pT3 was observed.

Aim of this analysis was to report on termination rates of the subgroup of patients treated with AS including different grade groups. The log-rank test was used to compare the time-to-reclassification between patients with GG1 and patients with GG2 tumors. Kaplan-Meier curves were produced using R, Version 3.6.

\section{Results}

Currently, no data on the primary endpoint of cancer-specific survival are available, implying that no randomized comparisons can be made. Accordingly, the results are shown for the population of patients actually treated with AS only as exploratory analysis.

\section{Intention-to-treat (ITT) population}

Of the 345 patients randomized to the study, 130 (42\%) were treated with AS (intention-to-treat population). As shown in Fig. 1, 10 patients did not accept randomization or switched to other options, and 21 patients changed from other randomization arms to the AS arm.

\section{As-treated (AT) population}

Finally, 141 patients were treated with AS according to the trial protocol. For 130 of these patients, complete followup data were available with a median follow-up time of 18.8 months (range 1-48 months) (Fig. 1, Table 2).

\section{Termination of AS and histological reclassification}

After a median follow-up time of 18.8 months, $56 \%(n=73)$ of the 130 patients terminated AS.

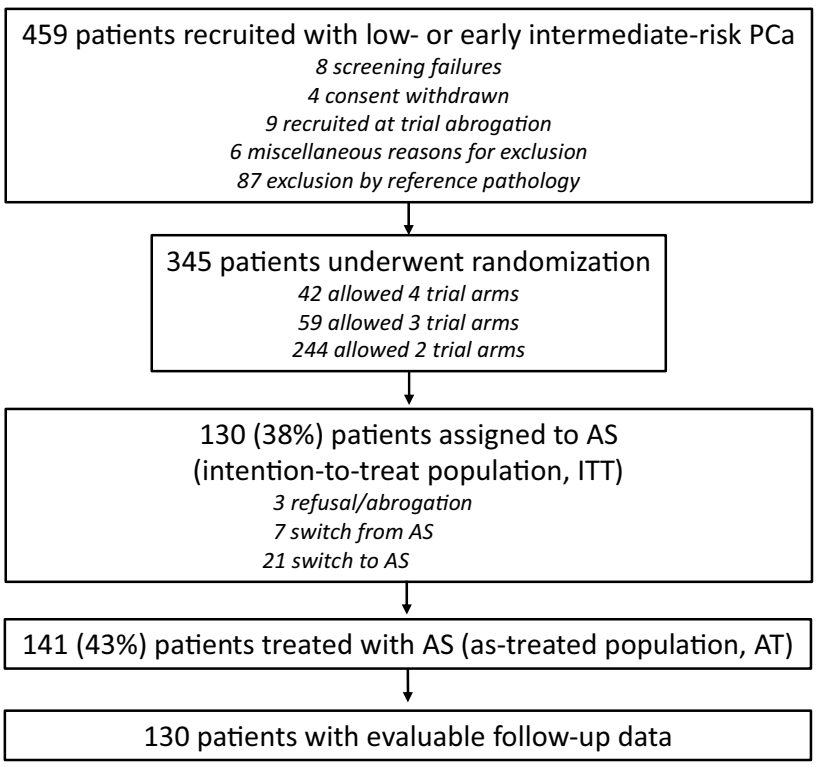

Fig. 1 CONSORT table

In 56 of these 73 patients (77\%), a histological reclassification, as defined above, was observed at the time of rebiopsy. Of the 56 patients with histological reclassifications, $31(55 \%)$ and $25(45 \%)$ had GG1 and GG2 prostate cancer at the initial biopsy, respectively. Of all 130 as-treated patients treated with AS, the GG at the first biopsy was GG1 and GG2 in 68\% $(n=88)$ and $32 \%(n=42)$ of the patients, respectively.

Histological reclassification occurred at a median time of 22.3 months after the first biopsy. Histological reclassification was observed in 39 (70\%) and $13(23 \%)$ patients in the first year and second or more years, respectively.

The time to histological reclassification differed significantly between patients with biopsy results of GG1 and GG2 ( $p=0.003$, see Fig. 2). At 12 months, $24 \%$ and $52 \%$ of the GG1 and GG2 patients had histological reclassification, respectively. At 24 months, $35 \%$ and $60 \%$ of the GG1 and GG2 patients had histological reclassification, respectively. A total of 37 of the $56(66 \%)$ specimens from patients with histological reclassification were available for and confirmed by reference pathology.

AS was terminated for various other reasons in 17 patients (13\%): psychological problems $(n=5)$, PSA elevation without histological confirmation of reclassification $(n=4)$, increasing voiding problems $(n=1)$, refusal to undergo rebiopsies $(n=3)$, and unknown reasons $(n=4)$.

The rebiopsy results, which were reported as the cause of AS termination, showed that $17 \%$ of the samples were GG3 and $13 \%$ were $\mathrm{GG} 4$ cancer.

In multivariate analysis, only ISUP grade group and prebiopsy PSA were predictive of reclassification (see Table 3). 
Table 2 Baseline characteristics

\begin{tabular}{lllll}
\hline & Total sample & Grade group 1 & Grade group 2 & $p$ value \\
\hline Gleason 7a $^{\mathrm{a}}$ & $n=42(32.3 \%)$ & - & - & - \\
PSA 6-10 $^{\mathrm{a}}$ & $n=85(65.4 \%)$ & $n=55(62.5 \%)$ & $n=30(71.4 \%)$ & 0.32 \\
Number of positive biopsies $^{\mathrm{b}}$ & $2(1-12)$ & $2(1-9)$ & $2(1-12)$ & 0.149 \\
Age [years] $^{\mathrm{b}}$ & $65(48-75)$ & $64.5(48-75)$ & $69(53-75)$ & 0.042 \\
Size of prostate $(n=113)^{\mathrm{b}}$ & $38.0(17-71)$ & $37.5(17-69)$ & $38.0(17-71)$ & 0.47 \\
Erectile dysfunction $(n=127)^{\mathrm{c}}$ & & & & 0.100 \\
Grade 0 (not present) & $92(72.4 \%)$ & $66(75.9 \%)$ & $26(65.0 \%)$ & \\
Grade 1 & $23(18.1 \%)$ & $15(17.2 \%)$ & $820.0 \%)$ & \\
Grade 2 & $7(5.5 \%)$ & $4(4.6 \%)$ & $3(7.5 \%)$ & \\
Grade 3 & $5(3.9 \%)$ & $2(2.3 \%)$ & $3(7.5 \%)$ & \\
Urinary incontinence $(n=129)^{\mathrm{d}}$ & & & & \\
Grade 0 (not present) & $126(97.7 \%)$ & $85(97.7 \%)$ & $41(97.6 \%)$ & \\
Grade 1 & $3(2.3 \%)$ & $2(2.3 \%)$ & $1(2.4 \%)$ & \\
\hline
\end{tabular}

${ }^{\mathrm{a}}$ Chi-Square test, ${ }^{\mathrm{b}}$ Mann-Whitney test, ${ }^{\mathrm{c}} \mathrm{Chi}$-Square test for trend, ${ }^{\mathrm{d}}$ Exact test of fisher

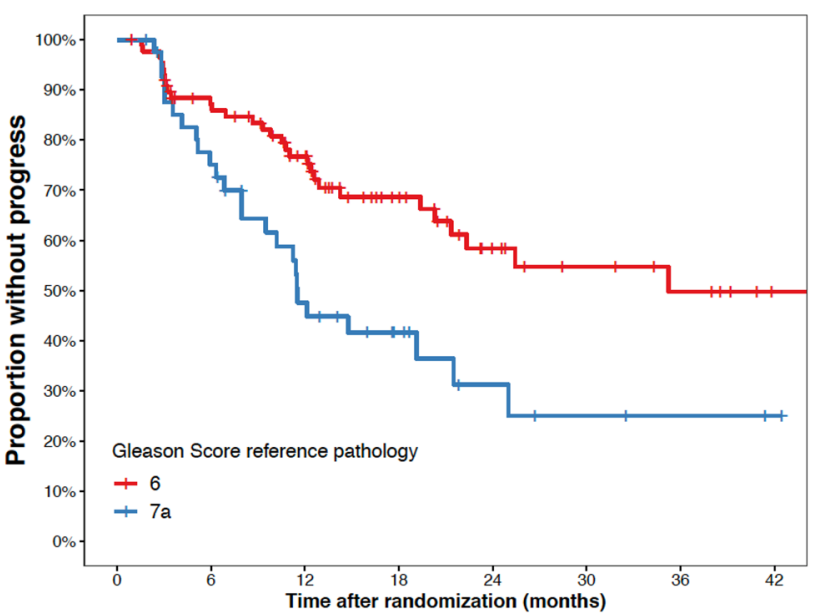

\begin{tabular}{|c|c|c|c|c|c|c|c|c|}
\hline \multicolumn{9}{|c|}{ No. at Risk } \\
\hline 6 & 88 & 70 & 54 & 31 & 18 & 13 & 10 & 5 \\
\hline $7 a$ & 42 & 30 & 17 & 10 & 5 & 3 & 2 & 1 \\
\hline
\end{tabular}

Fig. 2 Progression-free survival of patients with active surveillance (AS) in the PREFERE trial (as-treated population) (patients with GS 6 (red) versus GS 7a (blue) tumors). Gleason 6: 31 events; Gleason $7 \mathrm{a}: 25$ events

\section{Adherence to follow-up}

The adherence to the rebiopsy plan dropped to $57 \%$ at 6 months, $60 \%$ at 12 months, and $38 \%$ at 24 months. In all, $11 / 141(8 \%)$ patients had no follow-up documentation. In $15 \%, 8 \%$ and $8 \%$ of the patients, at least one, two, and three to nine follow-up visits were not registered, respectively.

Within 24 months, $75 \%$ of the rebiopsies were performed as requested; however, only $60 \%$ of the biopsies were performed at the requested time after the first biopsy.

\section{Active treatment after termination of AS}

In 48 of the 73 patients (66\%), active treatments after the termination of AS were reported (33 with radical prostatectomy, 11 with radiotherapy, 4 with LDR-brachytherapy).

In total, $60 \%$ and $40 \%$ of the patients who underwent radical prostatectomy (RP) after terminating AS and had histological reclassification were found to have GG1 and GG2 tumors in the radical prostatectomy specimen, respectively. No patients had radical prostatectomy specimen with tumor grades $\geq \mathrm{GG} 3$.

\section{Discussion}

The noninterventional treatment of prostate cancer, commonly termed "watchful waiting" (WW), was found to be inferior to RP with regard to long-term tumor-specific and overall survival [12]. The concept of AS aims at delaying or completely avoiding treatment through the timely detection of tumor progression. However, reliable evidence from randomized clinical trials corroborating the curative potential of AS does not exist. In particular, it is difficult to suitably select AS patients based on clinical criteria. Approximately, 20 years ago, expectant management strategies were implemented by Klotz et al. [13], tested in a consecutive cohort and finally published with a median follow-up of approximately 8 years. More than 10,000 patients have been reported to be managed with AS [2]. The only evidencecontaining data on an active monitoring strategy against active treatment options are available from the ProtecT trial [6]. However, compared to the SPCG-4 trial about the WW strategy, the reported series features a follow-up of all AS patients that is too short to assess long-term efficacy. The PREFERE trial is the second multicenter trial to 
Table 3 Prognostic factor analysis for histological reclassification (univariate and multivariate analysis)

\begin{tabular}{|c|c|c|c|c|c|c|}
\hline \multirow[t]{2}{*}{ Variable } & \multicolumn{3}{|l|}{ Univariate } & \multicolumn{3}{|l|}{ Multivariate } \\
\hline & Hazard ratio & $95 \% \mathrm{CI}$ & $p$ value & Hazard ratio & $95 \% \mathrm{CI}$ & $p$ value \\
\hline Gleason 7a vs. 6 & 2.22 & $1.30-3.78$ & 0.003 & 2.19 & $1.28-3.74$ & 0.004 \\
\hline PSA $6-10$ vs. $0-5$ & 2.12 & $1.13-3.95$ & 0.019 & 2.09 & $1.12-3.90$ & 0.021 \\
\hline Number pos. biopsies & 1.11 & $0.95-1.29$ & 0.21 & - & - & - \\
\hline Age [years] & 1.00 & $0.96-1.04$ & 0.95 & - & - & - \\
\hline Size of prostate $(n=113)$ & 0.99 & $0.97-1.02$ & 0.56 & - & - & - \\
\hline Erectile dysfunction $(n=127)$ & 1.08 & $0.77-1.51$ & 0.66 & - & - & - \\
\hline Urinary incontinence $(n=129)$ & 2.22 & $0.69-7.12$ & 0.18 & - & - & - \\
\hline
\end{tabular}

Note the high hazard ratio (2.22) for presence of urinary incontinence. There were only three patients with urinary incontinence, each of which showed histological reclassification. However, due to the small number of patients, the power of the related test of significance was very small prospectively assess a specifically designed AS strategy in a randomized comparative trial against other options to treat prostate cancer and included patients with not only low-risk but also early intermediate-risk profiles.

The most commonly used surrogate endpoint for evaluating the success of AS strategies is the rate of termination due to histological reclassification. Precisely, this endpoint was used in the PREFERE study. The termination of AS in the PREFERE trial was not based on PSA progression alone, as is common practice in most AS strategies and in clinical routine. In addition, there were several other factors that triggered the decision of termination of AS, such as patient discomfort, increasing PSA values, psychological problems or refusal to undergo rebiopsies. In this trial, the calculated 2-year rate of switching from AS to curative treatment was twice as high as that reported in recently published large series such as the PRIAS, ProtecT and the Klotz data [6, 12, 13] (see Table 4).

In the PRIAS trial, 415 of 2494 men (16.6\%) were reclassified based on repeat biopsies [14]. This may be explained by the more restrictive inclusion criteria: only patients with GG1 cancers and a maximum of two positive cores of at least eight cores from systematic biopsy were allowed. Therefore, even if early intermediate patients were excluded, the histological reclassification rate of true low-risk patients in the PREFERE trial was 35\%, which is twice as high as in the PRIAS trial. This may be explained by the following points: (1) sampling error at the time of the first biopsy; (2) two, instead of only one, recommended confirmation rebiopsies within the first 2 years of the PREFERE trial; and (3) different selection criteria.

In the ProtecT trial, $23 \%$ of the 545 patients were included in active monitoring with early intermediate or intermediate and higher risk classifications $(\mathrm{GG} \geq 3)$ [6]. The trigger for switching from the intervention to active treatment in the ProtecT trial was a $50 \%$ increase relative to the previous PSA value. At 2 years, the Protec $T$ trial found an active treatment rate in the AS population of less than $20 \%$. This may be caused by less frequent rebiopsies compared to the PRIAS and PREFERE trials.

In the Klotz series, 993 patients had been consecutively included in an active surveillance strategy since 1995. With a mean follow-up period $<10$ years, the cancer-specific survival rate was $98.5 \%$. The inclusion criteria and follow-up strategies were comparable to those of the PREFERE trial. In comparison to the $35 \%$ of the PREFERE trials, Klotz et al. [13] reported a very low rate of true histological reclassification which finally led to termination (see Table 4). This even larger difference towards lower rates of histological reclassification in the Klotz trial compared to that in the PREFERE trial may be due to only $13 \%$ of all tumors being GG2/3 tumors, the inclusion of patients with TUR-P sampling only and a missing rebiopsy at 24 months.

From the ProtecT trial, it is known that the 10-year cancer-specific survival rate is $98.8 \%$ for patients treated with active monitoring. However, the clinical progression rate
Table 4 Histological reclassification of patients in active surveillance trials

\begin{tabular}{lrrrlll}
\hline Author & Patients & \multicolumn{2}{l}{ ISUP grade } & Termination of AS & $\begin{array}{l}\text { Follow-up } \\
\text { (years) }\end{array}$ & $\begin{array}{l}\text { Histological reclas- } \\
\text { sification with repeat } \\
\text { biopsies }\end{array}$ \\
\cline { 2 - 5 } & & $1(\%)$ & $2(\%)$ & & 1.6 & $415 / 2494(16.6 \%)$ \\
\hline Bul et al. [9] & 2494 & 100 & & $527(21 \%)$ & 6.4 & $40 / 993(4 \%)$ \\
Klotz et al. [13] & 993 & 84 & 13 & $267(27 \%)$ & 10 & n.a \\
Hamdy et al. [6] & 545 & 77 & & $291(54 \%)$ & 1.6 & $56 / 130(40 \%)$ \\
Current date & 130 & 67 & 33 & $73(56 \%)$ & &
\end{tabular}


(e.g., metastasis, tumor-related obstruction) in this population at 10 years was $20.6 \%$ and a trend to an inferior survival has been already observed in an updated analysis [15]. Therefore, the cancer-specific survival data of this population compared to those of patients treated with surgery and radiotherapy will most likely decline over time [6]. In the PREFERE trial, 30\% of patients had GG 3/4 histology at the time of rebiopsy, leading to discontinuation of AS. On one hand, this highlights differences in the correct evaluation of prostate histology by biopsies. On the other hand, this clearly advocates for the early detection of possibly aggressive variants at an early stage with more frequent follow-up visits, including confirmation biopsies in the first 2 years of AS. Patients in AS are always afraid of being diagnosed too late at the time of AS discontinuation. This is especially true for patients with early intermediate risk. This study suggests that frequent rebiopsies are necessary to prevent undetected high-risk GG tumors in these patients.

The overall histological reclassification rates of $35 \%$ and $60 \%$ of low-risk and early intermediate-risk patients treated with AS, respectively, still reveals problems in the correct initial stratification of prostate cancer patients for AS. Recently, data for multiparametric magnetic resonance imaging (mpMRI) at the time of inclusion in an AS strategy have gained importance [16]. Guidelines in Europe have incorporated mpMRI in the primary diagnosis of prostate cancer [17].

In addition, the high frequency of follow-up visits for future AS strategies must include mpMRIs, which are compared to an initial mpMRI at the time of inclusion in AS [18]. In the current trial, MRI was used in only three patients for initial staging, so it was not evaluable.

A prospective trial in Germany has started to assess the effect of mpMRIs substituting repeat biopsies in the first 2 years of monitoring in AS (PROMM-AS, NCT03979573).

In summary, the subgroup analysis of patients treated with AS in the PREFERE trial showed that offering AS for early intermediate-risk patients presumably entails a high risk of early histological reclassification. Even when restricted to patients with GG1 tumors, the 35\% termination rate due to histological reclassification in the PREFERE trial is higher than expected. The main reasons for this difference may be suboptimal primary conventional staging and a much higher frequency of follow-up visits, including biopsies within the first 2 years, which led to histological reclassifications.

The limitations of this subgroup analysis of the PREFERE trial are the exploratory design of the analysis, the small number of patients with AS analyzed, the small number of radical prostatectomy samples for reference pathology, the early termination of the trial and the only intermediate follow-up time with lacks in follow-up data. The strengths are the data analysis of a subcohort of patients in a randomized trial, the high adherence to frequent rebiopsies and reference pathology.

\section{Conclusion}

In summary, the subgroup analysis of patients treated with AS within the PREFERE trial showed that offering AS for patients GG1 and GG2 prostate cancer based on conventional staging entails a high risk of early reclassification. Two out of three patients with GG2 tumors terminated AS due to histological reclassification.

This observation may lead to caution in offering AS to patients with intermediate-risk cancers, and these patients may be offered AS in carefully conducted clinical trials only. AS strategies should not further rely on conventional staging but should initially include mpMRI staging.

Acknowledgements Open Access funding provided by Projekt DEAL. The following departments of urology and/or radiation oncology have participated in the PREFERE study (in the order of patients recruited): Klinikum Leverkusen, Urologisches Zentrum Lübeck, Klinikum Lippe Detmold, Vinzenzkrankenhaus Hannover, Universitätsklinik des Saarlandes, Universitätsklinikum Carl Gustav Carus Dresden, Klinikum Gütersloh, Urologische Klinik München, St. Antonius-Hospital Eschweiler, Universitätsklinik Düsseldorf, SRH Wald-Klinikum Gera, Universitätsklinikum Hamburg-Eppendorf, Klinikum Hann. Münden, Johannes Wesling Klinikum Minden, Carl-Thiem-Klinikum Cottbus, Universitätsklinikum Köln, Klinikum Herford, Imland Klinik Rendsburg, Universitätsklinikum Würzburg, Klinikum Westfalen Dortmund, HELIOS Klinikum Erfurt, Städtisches Klinikum Karlsruhe, Städtisches Klinikum Lüneburg, Universitätsklinik Magdeburg, Klinikverbund Südwest Kliniken Nagold, Universitätsklinik Rostock, Klinikum Salzgitter, Krankenhaus der Barmherzigen Brüder Trier, Universitätsklinikum Ulm, Klinikum Wolfsburg, Klinikum am Bruderwald Bamberg, Vivantes Klinikum Am Urban Berlin, Charité Campus Benjamin Franklin, BG Klinikum Unfallkrankenhaus Berlin, Klinikum Fulda, SLK Kliniken Heilbronn-Klinikum am Gesundbrunnen, Klinikum Oldenburg, Marienhospital Wesel, Helios Klinikum Wuppertal, Klinikum Aschaffenburg, Marien-Krankenhaus Bergisch-Gladbach, Städtisches Klinikum Braunschweig, Zeisigwaldkliniken Bethanien Chemnitz, Alfried Krupp Krankenhaus Essen, Universitätsklinikum Freiburg, Klinikum der Ernst-Moritz-Arndt-Universität Greifswald, AMEOS Klinikum Haldensleben, Universitätsklinikum Heidelberg, Städtisches Krankenhaus Kiel, St.-Franziskus-Hospital Lohne, Klinikum Stuttgart Katharinenhospital, Bundeswehrkrankenhaus Ulm, Klinikum Nordoberpfalz, Standort Klinikum Weiden, Klinikum Darmstadt, Krankenhaus Nordwest Frankfurt, Universitätsmedizin Göttingen Georg-August-Universität, Heilig Geist-Krankenhaus Köln, Klinikum der Stadt Ludwigshafen, Marien-Hospital Marl, Kliniken Maria Hilf Mönchengladbach, Universitätsklinikum Münster, Clemenshospital Münster, Universitätsklinik Tübingen, Dr.-Horst-Schmidt-Kliniken Wiesbaden, Universitätsklinikum Mannheim, Evangelisches und Johanniter Klinikum Oberhausen, Jung-Stilling-Krankenhaus Siegen, Johanniter-Krankenhaus Genthin-Stendal, Missionsärztliche Klinik Würzburg.

Author contributions PA, TW, HS, RB-M, GK, MS: protocol/project development, data collection or management, data analysis, manuscript writing/editing; $\mathrm{MH}$ : protocol/project development, data analysis, manuscript writing/editing; PM, CM, SW: protocol/project development, 
data collection or management, data analysis (statistical analysis), manuscript writing/editing; KG, PR, MB, FS: data collection, manuscript writing/editing.

Funding The PREFERE study was funded by the German Cancer Aid (Deutsche Krebshilfe) and a consortium of German insurance companies.

\section{Compliance with ethical standards}

Conflict of interest Regarding the content of this paper, the authors declare that they have no conflict of interest.

Ethical approval All procedures performed in studies involving human participants were in accordance with the ethical standards of the institutional and/or national research committee (IRB approval by the ethics committee of the Ärztekammer Saarland in Homburg / Saar, Nr. 184/12) and with the 1964 Helsinki Declaration and its later amendments or comparable ethical standards. The Sponsor of the study was the German Cancer Society, Sponsor Number 2011-PCA-01.

Informed consent Informed consent was obtained from all individual participants included in the study.

Open Access This article is licensed under a Creative Commons Attribution 4.0 International License, which permits use, sharing, adaptation, distribution and reproduction in any medium or format, as long as you give appropriate credit to the original author(s) and the source, provide a link to the Creative Commons licence, and indicate if changes were made. The images or other third party material in this article are included in the article's Creative Commons licence, unless indicated otherwise in a credit line to the material. If material is not included in the article's Creative Commons licence and your intended use is not permitted by statutory regulation or exceeds the permitted use, you will need to obtain permission directly from the copyright holder. To view a copy of this licence, visit http://creativecommons.org/licenses/by/4.0/.

\section{References}

1. Robert Koch Institute, Germany. www.rki.de. Assessed 23 Nov 2019

2. Klotz L (2019) Contemporary approach to active surveillance for favorable risk prostate cancer. Asian J Urol 6:146-152

3. Ross HM, Kryvenko ON, Cowan JE et al (2012) Do adenocarcinomas of the prostate with gleason score (GS)_6 have the potential to metastasize to lymph nodes? Am J Surg Pathol 36:1346-1352
4. Eggener SE, Scardino PT, Walsh PC et al (2011) Predicting 15 -year prostate cancer specific mortality after radical prostatectomy. J Urol 185:869-875

5. Inoue LY, Trock BJ, Partin AW et al (2014) Modeling grade progression in an active surveillance study. Stat Med 33:930-939

6. Hamdy FC, Donovan JL, Lane JA et al (2016) 10-year outcomes after monitoring, surgery, or radiotherapy for localized prostate cancer. NEJM 375:1415-1424

7. Bill-Axelson A, Holmberg L, Garmo H et al (2014) Radical prostatectomy or watchful waiting in early prostate cancer. N Engl J Med 370:932-942

8. Cooperberg MR, Cowan JE, Hilton JF et al (2011) Outcomes of active surveillance for men with intermediate-risk prostate cancer. J Clin Oncol 29:228-234

9. Bul M, van den Bergh RCN, Zhu X et al (2012) Outcomes of initially expectantly managed patients with low or intermediate risk screen-detected localized prostate cancer. BJU Int 110:1672-1677

10. Ohlmann CH, Stöckle M, Albers P et al (2016) PREFERE—-study on the rise. Urologe A 55:313-317

11. Tokas T, Grabski B, Paul U et al (2018) A 12-year follow-up of ANNA/C-TRUS image-targeted biopsies in patients suspicious for prostate cancer. World J Urol 36:699-704

12. Bill-Axelson A, Holmberg L, Garmo H et al (2018) Radical prostatectomy or watchful waiting in prostate cancer-29-year followup. NEJM 379:2319-2329

13. Klotz L, Vesprini D, Sethukavalan P et al (2015) Long-term follow-up of a large active surveillance cohort of patients with prostate cancer. J Clin Oncol 33:272-277

14. Bul M, Zhu X, Valdagni R et al (2013) Active surveillance for low-risk prostate cancer worldwide: the PRIAS study. Eur Urol 63:597-603

15. Neal DE, Metcalfe C, Donovan JL et al (2019) Ten-year mortality, disease progression, and treatment-related side effects in men with localised prostate cancer from the ProtecT randomised controlled trial according to treatment received. Eur Urol. https:// doi.org/10.1016/j.eururo.2019.10.030 [Epub ahead of print]

16. Kasivisvanathan V, Rannikko AS, Borghi M et al (2018) MRItargeted or standard biopsy for prostate-cancer diagnosis. NEJM 378:1767-1777

17. EAU guideline for prostate cancer. www.uroweb.org. Assessed 23 Nov 2019

18. Fam MM, Yabes JG, Macleod LC et al (2019) Increasing utilization of multiparametric magnetic resonance imaging in prostate cancer surveillance. Urology 130:99-105

Publisher's Note Springer Nature remains neutral with regard to jurisdictional claims in published maps and institutional affiliations.

\section{Affiliations}

\section{Peter Albers ${ }^{1} \mathbb{D} \cdot$ Thomas Wiegel $^{2} \cdot$ Heinz Schmidberger $^{3} \cdot$ Roswitha Bussar-Maatz $^{4} \cdot$ Martin Härter $^{5}$. Glen Kristiansen ${ }^{6} \cdot$ Peter Martus $^{7} \cdot$ Christoph Meisner $^{7} \cdot$ Stefan Wellek $^{8} \cdot$ Klaus Grozinger $^{9} \cdot$ Peter Renner $^{10}$. Martin Burmester $^{11} \cdot$ Fried Schneider $^{12} \cdot$ Michael Stöckle $^{13}$}

Thomas Wiegel

thomas.wiegel@uniklinik-ulm.de

Heinz Schmidberger

heinz.schmidberger@unimedizin-mainz.de
Roswitha Bussar-Maatz

bussar-maatz@krebsgesellschaft.de

Martin Härter

m.haerter@uke.de 
Glen Kristiansen

glen.kristiansen@ukb.uni-bonn.de

Peter Martus

peter.martus@med.uni-tuebingen.de

Christoph Meisner

christoph.meisner@med.uni-tuebingen.de

Stefan Wellek

welleks@uni-mainz.de

Klaus Grozinger

grozinger@klinikum-lev.de

Peter Renner

renner@uz-luebeck.de

Martin Burmester

burmester@vincenzkrankenhaus.de

Fried Schneider

fried.schneider@klinikum-lippe.de

Michael Stöckle

michael.stoeckle@uks.eu

1 Department of Urology, University Hospital Düsseldorf, Düsseldorf, Germany

2 Department of Radiotherapy and Radiation Oncology, University Hospital Ulm, Ulm, Germany
3 Department of Radiotherapy and Radiation Oncology, University Hospital Mainz, Mainz, Germany

4 PREFERE Project Management, German Cancer Society, Berlin, Germany

5 Department of Medical Psychology, University Medical Center Hamburg-Eppendorf, Hamburg, Germany

6 Institute of Pathology, University Hospital Bonn, Bonn, Germany

7 Department of Biometry, University Hospital Tübingen, Tübingen, Germany

8 Institute of Medical Biostatistics, Epidemiology and Informatics, University Medical Center, Johannes Gutenberg University Mainz, Mainz, Germany

9 Department of Urology, Klinikum Leverkusen, Leverkusen, Germany

10 Department of Urology, Urologisches Zentrum, Lübeck, Germany

11 Department of Urology, Vincenz Krankenhaus, Hannover, Germany

12 Department of Urology, Klinikum Lippe, Detmold, Germany

13 Department of Urology, University Hospital Homburg/Saar, Homburg, Germany 